Pacific

Journal of

Mathematics

MELNIKOV FUNCTIONS FOR PERIOD ANNULUS, NONDEGENERATE CENTERS, HETEROCLINIC AND HOMOCLINIC CYCLES

Weigu Li, Jaume Llibre, and Xiang Zhang 


\title{
MELNIKOV FUNCTIONS FOR PERIOD ANNULUS, NONDEGENERATE CENTERS, HETEROCLINIC AND HOMOCLINIC CYCLES
}

\author{
Weigu Li, Jaume Llibre, And Xiang Zhang
}

\begin{abstract}
We give sufficient conditions in terms of the Melnikov functions in order that an analytic or a polynomial differential system in the real plane has a period annulus.

We study the first nonzero Melnikov function of the analytic differential systems in the real plane obtained by perturbing a Hamiltonian system having either a nondegenerate center, a heteroclinic cycle, a homoclinic cycle, or three cycles obtained connecting the four separatrices of a saddle. All the singular points of these cycles are hyperbolic saddles.

Finally, using the first nonzero Melnikov function we give a new proof of a result of Roussarie on the finite cyclicity of the homoclinic orbit of the integrable system when we perturb it inside the class of analytic differential systems.
\end{abstract}

\section{Introduction and statement of the main results.}

We consider the planar vector fields $\mathcal{X}_{\epsilon}$ associated to the system:

$$
\begin{aligned}
& \dot{x}=X(x, y, \lambda, \epsilon)=p(x, y)+\epsilon P(x, y, \lambda, \epsilon), \\
& \dot{y}=Y(x, y, \lambda, \epsilon)=q(x, y)+\epsilon Q(x, y, \lambda, \epsilon),
\end{aligned}
$$

where $X, Y$ depend analytically on their variables and parameters $\lambda \in \Lambda$, and $\epsilon \in \mathbf{R}, \Lambda \subset \mathbf{R}^{r}$ is an open region. Assume that for $\epsilon=0$, system (1) has a period annulus; i.e., a continuous family of periodic orbits. As usual, the dot denotes derivative with respect to the time variable $t$. We say that system (1) with $\epsilon=0$ is the unperturbed system, while system (1) with $\epsilon \neq 0$ is the perturbed one.

Given any compact subset $\mathbf{D}$ of $\Lambda$ and $\epsilon_{0}>0$ small, we assume that there is a transversal section $J$ to the vector fields $\mathcal{X}_{\epsilon}$ in the region covered by the period annulus for $|\epsilon|<\epsilon_{0}$ and $\lambda \in \mathbf{D}$. Let $u$ be an analytical parameterization of $J$. Then there is a subsection $\Sigma \subset J$ such that the Poincaré return map $(u, \lambda, \epsilon) \mapsto \Pi(u, \lambda, \epsilon)$ is defined from $\Sigma \times \mathbf{D} \times\left(-\epsilon_{0}, \epsilon_{0}\right)$ to $J$. The displacement function $d(u, \lambda, \epsilon)$ is defined as $d(u, \lambda, \epsilon)=\Pi(u, \lambda, \epsilon)-$ $u$. Since system (1) has a period annulus for $\epsilon=0$, we have $d(u, \lambda, 0) \equiv 0$, 
and thus for $u \in \Sigma, \lambda \in \mathbf{D}$ and $\epsilon_{0}>0$ sufficiently small, we have

$$
d(u, \lambda, \epsilon)=\sum_{i=1}^{\infty} M_{i}(u, \lambda) \epsilon^{i}
$$

The function $M_{i}$ is called the $i$-th Melnikov function. In what follows the notation $|\epsilon| \ll 1$ means for all $\epsilon$ such that $|\epsilon|<\epsilon_{0}$ with $\epsilon_{0}>0$ sufficiently small. The first part of this paper is dedicated to period annulus.

Theorem 1. For any compact set $\mathbf{D} \subset \Lambda, \epsilon_{0}>0$ and a transversal section $\Sigma$ for which the displacement function (2) is defined, there exists a natural number $N$ depending on $\mathbf{D}$ such that for any $\lambda_{0} \in \mathbf{D}$, if $M_{i}\left(u, \lambda_{0}\right) \equiv 0$, for $u \in \Sigma, 1 \leq i \leq N$, then system (1) has a period annulus for $\lambda=\lambda_{0}$ and $|\epsilon| \ll 1$.

Theorem 2. Assume that

$$
\begin{aligned}
& P(x, y, \lambda, \epsilon)=P(x, y, \epsilon)=\sum_{i=0}^{l} P_{i}(x, y) \epsilon^{i} \\
& Q(x, y, \lambda, \epsilon)=Q(x, y, \epsilon)=\sum_{i=0}^{l} Q_{i}(x, y) \epsilon^{i}
\end{aligned}
$$

and $P_{i}(x, y), Q_{i}(x, y)$ are polynomials in the variables $x$ and $y$ of degree at most $n$, then there exists a natural number $N$ depending on the unperturbed system $\mathcal{X}_{0}$ and on the natural numbers $l, n$ such that if $M_{i}(u) \equiv 0$ for $1 \leq$ $i \leq N$, then system (1) has a period annulus for $|\epsilon| \ll 1$.

The second part of this paper is concerned with the properties of Melnikov functions near a nondegenerate center and a hyperbolic heteroclinic or homoclinic cycle for the perturbed Hamiltonian systems.

We first recall some definitions. Let $\mathcal{X}$ be a vector field in the plane. A center is a singular point of $\mathcal{X}$ for which there is a neighbourhood filled of periodic orbits with the exception of the singular point. A center of $\mathcal{X}$ is called nondegenerate if it has a pair of pure imaginary eigenvalues. A heteroclinic cycle $\Gamma$ for $\mathcal{X}$ is a finite collection of separatrices of hyperbolic sectors $\gamma_{1}, \gamma_{2}, \ldots, \gamma_{n}$ and a finite collection of singular points $p_{1}, p_{2}, \ldots, p_{n}$ such that the $\alpha$-limit set of $\gamma_{i}$ is $p_{i}$ for $i=1, \ldots, n$, the $\omega$-limit set of $\gamma_{i}$ is $p_{i+1}$ for $i=1,2, \ldots, n-1$ and the $\omega$-limit set of $\gamma_{n}$ is $p_{1}$. Moreover, some of the $p_{i}$ can be repeated. A heteroclinic cycle $\Gamma$ is called hyperbolic, if all its singular points are hyperbolic saddles. A heteroclinic cycle becomes a homoclinic one, if it consists of one singular point and one separatrix. Now 
we consider the following perturbed Hamiltonian system:

$$
\begin{aligned}
& \dot{x}=\frac{\partial H(x, y)}{\partial y}+\epsilon P(x, y, \epsilon), \\
& \dot{y}=-\frac{\partial H(x, y)}{\partial x}+\epsilon Q(x, y, \epsilon),
\end{aligned}
$$

where $H, P, Q$ are analytical functions in the variables $(x, y) \in \mathbf{R}^{2}$ and in the parameter $\epsilon \in(\mathbf{R}, 0)$. Here $(\mathbf{R}, 0)$ denotes a small neighbourhood of zero, and $C^{\omega}(\mathbf{R}, 0)$ denotes the set of analytic functions in a small neighbourhood of zero. In this case, as usual, we parameterize the transversal section $J$ by the Hamiltonian constant $h=H$. We assume that the unperturbed Hamiltonian system has a continuous family of periodic orbits $\gamma_{h} \subset H^{-1}(h)$ for $0<h \ll$ 1.

Theorem 3. For system (3) assume that when $h \searrow 0, \gamma_{h} \rightarrow(0,0)$, a nondegenerate center of the unperturbed system. Then the following hold:

(1) $M_{1}(h)$ can be analytically continued to $h=0$, and

$$
M_{1}(0)=0, \quad M_{1}^{\prime}(0)=\left.\frac{2 \pi}{\beta}\left(\frac{\partial P}{\partial x}+\frac{\partial Q}{\partial y}\right)\right|_{(x, y, \epsilon)=(0,0,0)},
$$

where $\pm i \beta$ with $\beta>0$ are the eigenvalues of the center.

(2) If $M_{i}(h) \equiv 0$ for $1 \leq i \leq k-1$, then $M_{k}(h)$ can be analytically continued to $h=0$ and $M_{k}(0)=0$.

Theorem 4. For system (3) assume that when $h \searrow 0, \gamma_{h} \rightarrow \gamma_{0}$, a heteroclinic cycle of the unperturbed system consisting of $n$ hyperbolic saddles $p_{1}, p_{2} \ldots, p_{n}$ (eventually they can be repeated) and the corresponding $n$ separatrices. Then the following hold:

(1) There exist analytical functions $a_{1}(h), b_{1}(h) \in C^{\omega}(\mathbf{R}, 0)$ such that

$$
M_{1}(h)=a_{1}(h)+b_{1}(h) \ln h, \quad 0<h \ll 1,
$$

with

$$
\begin{aligned}
& a_{1}(0)=\int_{\gamma_{0}} P(x, y, 0) d y-Q(x, y, 0) d x, \\
& b_{1}(0)=0 \\
& b_{1}^{\prime}(0)=-\left.\sum_{i=1}^{n} \frac{1}{\lambda_{i}}\left(\frac{\partial P}{\partial x}+\frac{\partial Q}{\partial y}\right)\right|_{(x, y)=p_{i}, \epsilon=0},
\end{aligned}
$$

where $-\lambda_{i}<0<\lambda_{i}$ are the eigenvalues of the saddle $p_{i}$. Moreover, if

$$
\left.\left(\frac{\partial P}{\partial x}+\frac{\partial Q}{\partial y}\right)\right|_{(x, y)=p_{i}, \epsilon=0}=0, \text { for } i=1, \ldots, n
$$


then

$$
a_{1}^{\prime}(0)=\left.\int_{\gamma_{0}}\left(\frac{\partial P}{\partial x}+\frac{\partial Q}{\partial y}\right)\right|_{\epsilon=0} d t .
$$

(2) If $n=1$, and $\gamma_{0}$ is a homoclinic cycle of a hyperbolic saddle, and $M_{i}(h) \equiv 0$ for $1 \leq i \leq k-1$, then there exist analytical functions $a_{k}(h), b_{k}(h) \in C^{\omega}(\mathbf{R}, 0)$ with $b_{k}(0)=0$, such that

$$
M_{k}(h)=a_{k}(h)+b_{k}(h) \ln h, \quad 0<h \ll 1 .
$$

In general, Statement (2) of Theorem 4 cannot be generalized to heteroclinic cycles with two saddles or more. Now we consider the so-called 8 -figure heteroclinic cycles, i.e., the cycles consisting of one saddle and its two homoclinic orbits. Assume that for $\epsilon=0$, system (3) has two homoclinic orbits $\gamma_{0}^{ \pm}$of a hyperbolic saddle, called the 8-figure cycle, and three families of periodic orbits: $\gamma_{h} \subset\left\{H^{-1}(h), h>0\right\}, \gamma_{h}^{+} \cup \gamma_{h}^{-} \subset\left\{H^{-1}(h), h<0\right\}$, such that $\gamma_{h} \rightarrow \gamma_{0}^{+} \cup \gamma_{0}^{-}$when $h \searrow 0$, and $\gamma_{h}^{ \pm} \rightarrow \gamma_{0}^{ \pm}$when $h \nearrow 0$, see Figure 1 . Three classes of Melnikov functions are defined corresponding to the three period annuli: $M_{k}(h), M_{k}^{ \pm}(h)$ for $k \geq 1$. Then we have:

Theorem 5. If $M_{i}=M_{i}^{+}=M_{i}^{-} \equiv 0$ for $0 \leq i \leq k-1$ with $k \geq 1$, then the following hold:

(1) If one of three functions $M_{k}, M_{k}^{+}, M_{k}^{-}$can be analytically continued to $h=0$, then the other two can also be continued.

(2) If two of three functions $M_{k}, M_{k}^{+}, M_{k}^{-}$are identically zero, then the third one is identically zero.

(3) There exist analytical functions $a_{k}(h), b_{k}(h) \in C^{\omega}(\mathbf{R}, 0)$ with $b_{k}(0)=0$ such that $M_{k}(h)=a_{k}(h)+b_{k}(h) \ln h$ for $0<h \ll 1$.
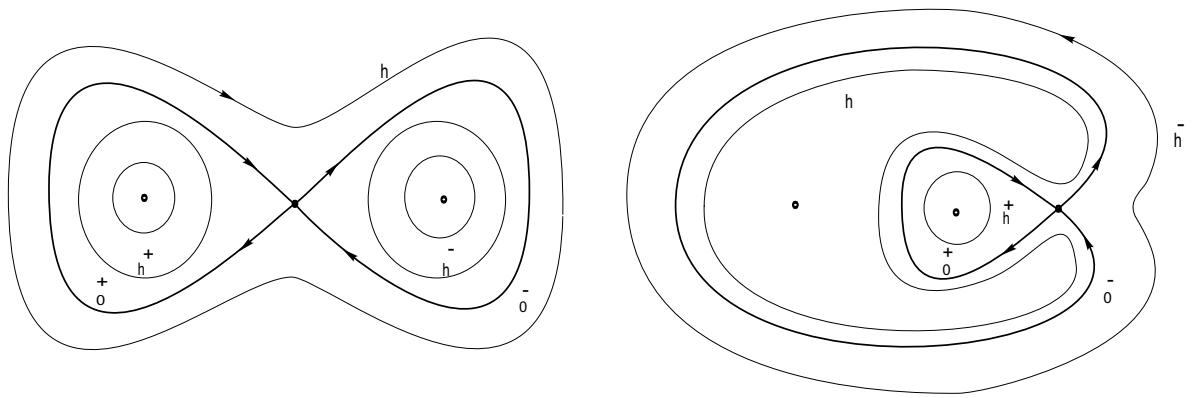

Figure 1. The two 8-figure heteroclinic cycles.

We remark that by using Melnikov functions we can determinate the cyclicity of a center or of a homoclinic cycle. Assume that the origin $(0,0)$ 
is a nondegenerate center of system (3) for $\epsilon=0$. Without loss of generality, we can assume that

$$
P(0,0, \epsilon)=Q(0,0, \epsilon) \equiv 0,
$$

which means that our perturbation preserves the singular point $(0,0)$ fixed. Let $f(h) \in C^{\omega}(\mathbf{R}, 0)$. If $f(h)=a h^{n}+O\left(h^{n+1}\right)$ with $a \neq 0$, we define $m(f)=n$. If $f \equiv 0$, we define $m(f)=\infty$.

Remark 6. Let the origin $(0,0)$ be a nondegenerate center of system (3) for $\epsilon=0$. Assume that (4) holds, and there exist integers $k \geq 1, m \geq 0$ such that

$$
M_{i}(h) \equiv 0 \text { for } i \leq k-1 \text {, and } m\left(M_{k}(h)\right)=n+1 \text { with } 0 \leq n<\infty .
$$

Then system (3) has at most $n$ (taking into account their multiplicity) limit cycles in some neighbourhood of the origin for $|\epsilon| \ll 1$.

Remark 7. Assume that when $h \searrow 0, \gamma_{h} \rightarrow \gamma_{0}$, where $\gamma_{0}$ is a homoclinic cycle of a hyperbolic saddle. Let $k \geq 1$ be such an integer that $M_{i}(h) \equiv 0$ for $0 \leq i \leq k-1$ and $M_{k}(h)=a_{k}(h)+b_{k}(h) \ln h$ is not identically zero, then there exists a neighbourhood $U$ of $\gamma_{0}$ such that for $|\epsilon| \ll 1$, system (3) in $U$ has at most $2 m\left(b_{k}\right)-1$ limit cycles if $m\left(a_{k}\right) \geq m\left(b_{k}\right)$; and $2 m\left(a_{k}\right)$ limit cycles if $m\left(a_{k}\right)<m\left(b_{k}\right)$, these estimates hold taken into account the multiplicity of the limit cycles.

We point out that Roussarie in [10] obtained the result of Remark 7 for $k=1$ and the method works for $k \geq 2$ also. As an application of Remark 7, we will give a simple proof of the finite cyclicity of the homoclinic cycle of infinite codimension under a one-parameter purterbation, which is a particular case of a result due to Roussarie [11]. First we recall that a homoclinic cycle $\gamma$ is said to be of infinite codimension if there exists a continuous family of periodic orbits tending to the cycle $\gamma$.

Theorem 8. Let $\mathcal{X}_{\epsilon}$ be an one parameter analytic family of planar vector fields. Assume that $\mathcal{X}_{0}$ has a homoclinic cycle $\gamma$ of a hyperbolic saddle of infinite codimension, then there exists a neighbourhood $U$ of $\gamma$ and a natural number $N$ such that $\mathcal{X}_{\epsilon}$ has at most $N$ limit cycles (taking into account their multiplicity) in $U$ for $|\epsilon| \ll 1$.

This paper is organized as follows: In Section 2, we prove Theorems 1 and 2. In Section 3 we first recall three important results, one is about the formula for computing Melnikov functions of arbitrary order, the other two are about the normalization of planar Hamiltonian vector fields near a nondegenerate center or a hyperbolic saddle, which are the main tools in this paper, and then prove Theorem 3. In Section 4 we prove Theorems 4 and 5. In Section 5 (after Proposition 25) we prove Theorem 8. 


\section{Proofs of Theorems 1 and 2 .}

Lemma 9. Let $d(u, \lambda, \epsilon)$ be the displacement function as defined in Section 1. Assume that, for $\lambda=\lambda_{0} \in \mathbf{D}, d\left(u, \lambda_{0}, \epsilon\right) \equiv 0$, then there exists a neighbourhood $U$ of $\lambda_{0}$ and a natural number $N$ such that for any $\lambda \in U$, if $M_{i}(u, \lambda) \equiv 0,1 \leq i \leq N$, then $M_{i}(u, \lambda) \equiv 0$ for all natural number $i$, i.e., system (1) has a period annulus for $|\epsilon| \ll 1$.

Proof. By the assumption, $M_{i}\left(u, \lambda_{0}\right) \equiv 0$ for all $i$. For $u_{0} \in \Sigma$, let

$$
M_{i}(u, \lambda)=\sum_{j=0}^{\infty} a_{j}^{i}\left(\lambda, u_{0}\right)\left(u-u_{0}\right)^{j} .
$$

Then $a_{j}^{i}\left(\lambda_{0}, u_{0}\right)=0, i \geq 1, j \geq 0$. Denote by $\mathcal{A}$ the ring of germs of analytic functions at $\lambda_{0}$ and $\mathcal{I}=\mathcal{I}\left\{\hat{a}_{j}^{i}\left(\cdot, u_{0}\right)\right\}_{i \geq 1, j \geq 0}$ the ideal generated by the germs of the analytical functions $a_{j}^{i}$ at $\lambda=\lambda_{0}$. Since the $\operatorname{ring} \mathcal{A}$ is Noetherian (see for instance $[\mathbf{3}]$, p. 161, Theorem 6.3 .3 ), and so $\mathcal{I}$ is generated by a finite number of germs $\hat{a}_{j}^{i}$ :

$$
\mathcal{I}=\mathcal{I}\left\{\hat{a}_{j_{1}}^{i_{1}}, \hat{a}_{j_{2}}^{i_{2}}, \ldots, \hat{a}_{j_{n}}^{i_{n}}\right\} .
$$

Let $N=\max \left\{i_{1}, i_{2}, \ldots, i_{n}\right\}$. Then there exist analytical functions $h_{i, k}(\lambda, u)$, $1 \leq k \leq N, i \geq 1$ defined in some neighborhood $U \times V$ of $\left(\lambda_{0}, u_{0}\right)$ such that

$$
M_{i}(u, \lambda)=\sum_{k=1}^{n} a_{j_{k}}^{i_{k}}\left(\lambda, u_{0}\right) h_{i, k}(\lambda, u) .
$$

(See [12], p. 79, Proposition 4.) For $\lambda \in U$, if $M_{i}(u, \lambda) \equiv 0,1 \leq i \leq N$, then

$$
a_{j}^{i}\left(\lambda, u_{0}\right)=0, \text { for } 1 \leq i \leq N, j \geq 0,
$$

which, by (5), implies $M_{i}(u, \lambda) \equiv 0$ for $i \geq 1$. The proof of Lemma 9 is complete.

Proof of Theorem 1. Let

$$
\begin{aligned}
D_{i} & =\left\{\lambda \in \mathbf{D} \mid \exists k, k \leq i \text { with } M_{k}(u, \lambda) \text { not identically vanishing }\right\} \\
D & =\left\{\lambda \in \mathbf{D} \mid M_{i}(u, \lambda) \equiv 0, \forall i>0\right\} .
\end{aligned}
$$

Then

$$
\mathbf{D}=\left(\bigcup_{i=1}^{\infty} D_{i}\right) \bigcup D .
$$

If the conclusion is not true, then there exists a sequence of parameter values $\lambda_{n} \in \mathbf{D}$ such that

$$
M_{i}\left(u, \lambda_{n}\right) \equiv 0 \text { for } 1 \leq i \leq n \text { and } d\left(u, \lambda_{n}, \epsilon\right) \text { is not identically zero. }
$$

By the compactness of $\mathbf{D}$, we can assume that $\lambda_{n} \rightarrow \bar{\lambda} \in \mathbf{D}$. Since $D_{i}$ are open subsets of $\mathbf{D}, \bar{\lambda} \in D$, which is a contradiction with Lemma 9 . 
Proof of Theorem 2. Let

$$
P_{i}(x, y)=\sum_{0 \leq j+k \leq n} p_{j, k}^{i} x^{j} y^{k}, \quad Q_{i}(x, y)=\sum_{0 \leq j+k \leq n} q_{j, k}^{i} x^{j} y^{k} .
$$

We consider the coefficients of the polynomials $p_{j, k}^{i}, q_{j, k}^{i}$ and $\epsilon$ as the parameters. Note that system (4) preserves unchanged under the parameter change $\epsilon \rightarrow \delta^{-1} \epsilon, p_{j, k}^{i} \rightarrow \delta^{i} p_{j, k}^{i}, q_{j, k}^{i} \rightarrow \delta^{i} q_{j, k}^{i}$. Therefore, we can assume that $\left|p_{j, k}^{i}\right| \leq 1,\left|q_{j, k}^{i}\right| \leq 1$. Hence Theorem 2 becomes a corollary of Theorem 1.

Example 1. For the quadratic perturbations of Bagdanov-Takens system (see $[4])$ :

$$
\begin{aligned}
& \dot{x}=y+\epsilon P(x, y) \\
& \dot{y}=-x-x^{2}+\epsilon Q(x, y)
\end{aligned}
$$

where $P, Q$ are polynomials of degree at most $2, N=4$.

Example 2. For the quadratic perturbation of quadratic Hamiltonian system which preserves the center fixed (see [7]):

$$
\begin{aligned}
& \dot{x}=\frac{\partial H(x, y)}{\partial y}+\epsilon P(x, y) \\
& \dot{y}=-\frac{\partial H(x, y)}{\partial x}+\epsilon Q(x, y)
\end{aligned}
$$

where $H$ is a polynomial of degree 3 , the origin $(0,0)$ is a center of the unperturbed system and $P, Q$ are polynomails of degree $\leq 2$ with $P(0,0)=$ $Q(0,0)=0, N=6$.

\section{Analyticity of Melnikov functions at a center.}

We first recall three results which are necessary in the proof of our theorems. The first one is about the computation of the Melnikov functions of system (3). We consider now the equivalent form of system (3):

$$
\omega_{\epsilon}=\left(\frac{\partial H}{\partial x}+\epsilon Q\right) d x+\left(\frac{\partial H}{\partial y}-\epsilon P\right) d y=0 .
$$

Let

$$
\omega_{\epsilon}=\sum_{i=0}^{\infty} \omega_{i} \epsilon^{i} .
$$

Then $\omega_{0}=d H$, and $\omega_{i}$ 's are analytical 1-form. The following result is due to Poggiale [9], its proof can be found in [12].

Proposition 10.

(1) $M_{1}(h)=-\int_{\gamma_{0}} \omega_{1}$; 
(2) If $M_{i}(h) \equiv 0$ for $1 \leq i \leq k$, then

$$
M_{k+1}(h)=\int_{\gamma_{h}}\left(\sum_{i=1}^{k} g_{i} \omega_{k-i+1}-\omega_{k+1}\right),
$$

where the analytic functions $g_{i}, i=1,2, \ldots, k$, are defined inductively by

$$
\omega_{i}-g_{i} d H=\sum_{j=1}^{i-1} g_{j} \omega_{i-j}+d R_{i} .
$$

The next two classical results are about the normalization of planar Hamiltonian system near a nondegenerate center or a hyperbolic saddle respectively (for the proofs, see, for instance, $[\mathbf{6}]$ and $[\mathbf{8}]$ ).

Consider now the following planar Hamiltonian system:

$$
\begin{aligned}
& \dot{x}=\frac{\partial H(x, y)}{\partial y}, \\
& \dot{y}=-\frac{\partial H(x, y)}{\partial x},
\end{aligned}
$$

where $H$ is an analytical function defined in some neighbourhood of the origin $(0,0)$.

Proposition 11. Assume that the origin $(0,0)$ is a nondegenerate center of system (7) with eigenvalues $\pm i \beta, \beta>0$, then there exist an analytical area-preserving transformation of variables: $(x, y)=G(u, v)$ in some neighbourhood of the origin and a function $f \in C^{\omega}(\mathbf{R}, 0)$ with $f(0)=0, f^{\prime}(0)=\frac{\beta}{2}$ such that $f\left(u^{2}+v^{2}\right)=H \circ G(u, v)$ and system (7) is changed to the form:

$$
\begin{aligned}
& \dot{u}=2 v f^{\prime}\left(u^{2}+v^{2}\right), \\
& \dot{v}=-2 u f^{\prime}\left(u^{2}+v^{2}\right) .
\end{aligned}
$$

Proposition 12. Assume that the origin $(0,0)$ is a hyperbolic saddle of system (7) with eigenvalues $\pm \lambda, \lambda>0$, then there exist an analytical areapreserving transformation of variables: $(x, y)=G(u, v)$ in some neighbourhood of the origin and a function $f \in C^{\omega}(\mathbf{R}, 0)$ with $f(0)=0, f^{\prime}(0)=\lambda$ such that $f(u v)=H \circ G(u, v)$ and system (7) is changed to the form:

$$
\begin{aligned}
& \dot{u}=u f^{\prime}(u v), \\
& \dot{v}=-v f^{\prime}(u v) .
\end{aligned}
$$

Lemma 13. Assume that $f \in C^{\omega}(\mathbf{R}, 0), f(0)=0, f^{\prime}(0)>0$, and $F \in$ $C^{\omega}\left(\mathbf{R}^{2}, \mathbf{0}\right)$. Define function

$$
M(h):=\iint_{f\left(x^{2}+y^{2}\right) \leq h} F(x, y) d x d y, \quad 0<h \ll 1 .
$$

Then the following statements hold: 
(1) $M(h)$ can analytically be continued to $h=0$, and

$$
M(0)=0, M^{\prime}(0)=\frac{\pi}{f^{\prime}(0)} F(0,0) .
$$

(2) If

$$
F(x, y)=\sum_{n=0}^{\infty} F_{n}(x, y), F_{n}(x, y)=\sum_{i=0}^{n} b_{i, n} x^{n-i} y^{i}
$$

then

$$
M(h) \equiv 0 \Longleftrightarrow C_{m}:=\sum_{k=0}^{m}(2 m-2 k-1) ! !(2 k-1) ! ! b_{2 k, 2 m}=0, \forall m \geq 0,
$$

where $(-1) ! !:=1$.

Proof. Assume that series (8) is convergent in the square $D=\{(x, y) \in$ $\left.\mathbf{C}^{2}|| x|\leq R| y \mid, \leq R\right\}$. Let $K=\sup _{D}|F|$. By Cauchy inequality, $\left|b_{2 k, 2 m}\right| \leq$ $K R^{-2 m}$. Let $d_{m}=\frac{C_{m}}{(2 m+2) ! !}$, then

$$
\left|d_{m}\right| \leq(m+1) K R^{-2 m},
$$

which implies the function $g(r):=\sum_{m=0}^{\infty} d_{m} r^{m+1}$ is analytic in the region $|r| \leq R^{2}$. Now we calculate the function $M(h)$. By introducing the polar coordinates $x=r \cos \theta, y=r \sin \theta, s=\sqrt{f^{-1}(h)}$, we have

$$
\begin{aligned}
M(h) & =\int_{0}^{s} d r \int_{0}^{2 \pi} r F(r \cos \theta, r \sin \theta) d \theta \\
& =\sum_{n=0}^{\infty} \int_{0}^{s} r^{n+1} d r \int_{0}^{2 \pi} \sum_{i=0}^{n} b_{i, n} \cos ^{n-i} \theta \sin ^{i} \theta d \theta \\
& =\sum_{m=0}^{\infty} \int_{0}^{s} r^{2 m+1} d r \int_{0}^{2 \pi} \sum_{i=0}^{2 m} b_{i, 2 m} \cos ^{2 m-i} \theta \sin ^{i} \theta d \theta \\
& =\sum_{m=0}^{\infty} \int_{0}^{s} r^{2 m+1} d r \int_{0}^{2 \pi} \sum_{k=0}^{m} b_{2 k, 2 m} \cos ^{2 m-2 k} \theta \sin ^{2 k} \theta d \theta \\
& =2 \pi \sum_{m=0}^{\infty} \sum_{k=0}^{m} \frac{(2 m-2 k-1) ! !(2 k-1) ! ! b_{2 k, 2 m}}{(2 m+2) ! !} s^{2 m+2} \\
& =2 \pi \sum_{m=0}^{\infty} d_{m}\left(f^{-1}(h)\right)^{m+1}=2 \pi g\left(f^{-1}(h)\right),
\end{aligned}
$$

which is analytical at $h=0$, and satisfies the following:

$$
M(0)=2 \pi g(0)=0, M^{\prime}(0)=2 \pi g^{\prime}(0)\left(f^{\prime}(0)\right)^{-1}=\frac{\pi}{f^{\prime}(0)} F(0,0) .
$$


In the computation above, we have used that

$$
\int_{0}^{2 \pi} \cos ^{2 m-2 k} \theta \sin ^{2 k} \theta d \theta=\frac{(2 m-2 k-1) ! !(2 k-1) ! !}{(2 m) ! !} .
$$

For a proof, see [2]. Statement (2) is obvious by noting that

$$
M(h) \equiv 0 \Longleftrightarrow g(r) \equiv 0 \Longleftrightarrow d_{m}=0, \forall m .
$$

Remark 14. Condition (9) is equivalent to

$$
\int_{0}^{2 \pi} F(r \cos \theta, r \sin \theta) d \theta \equiv 0,0 \leq r \ll 1 .
$$

Now we consider an analytical system

$$
\begin{aligned}
& \dot{u}=\frac{\partial H}{\partial v}, \\
& \dot{v}=-\frac{\partial H}{\partial u} .
\end{aligned}
$$

Lemma 15. Assume that system (12) has a family of periodic orbits $\gamma_{h}$ : $H(u, v)=h, 0<h<\bar{h}$. The origin $(0,0)=H^{-1}(0)$ is a nondegenerate center with eigenvalues $\pm i \beta$ with $\beta>0$. Let $\omega=-P(u, v) d v+Q(u, v) d u$ be an analytical 1-form defined in some neighbourhood of the origin, then the function $M(h):=\int_{\gamma_{h}} \omega$ can be analytically continued to $h=0$, and

$$
M(0)=0, \quad M^{\prime}(0)=\left.\frac{2 \pi}{\beta}\left(\frac{\partial P}{\partial u}+\frac{\partial Q}{\partial v}\right)\right|_{(u, v)=(0,0)} .
$$

Proof. By Proposition 11, there exist an area-preserving transformation

$$
u=u(x, y), v=v(x, y), u(0,0)=0, v(0,0)=0,
$$

and a function $f \in C^{\omega}(\mathbf{R}, 0)$ with $f(0)=0, f^{\prime}(0)=\frac{\beta}{2}$, such that

$$
H(u(x, y), v(x, y))=f\left(x^{2}+y^{2}\right) .
$$

Thus, by Green's formula, we obtain

$$
\begin{aligned}
M(h) & =\iint_{H(u, v) \leq h}\left(\frac{\partial P}{\partial u}+\frac{\partial Q}{\partial v}\right) d u d v \\
& =\iint_{f\left(x^{2}+y^{2}\right) \leq h} F(x, y) d x d y, \quad F(x, y)=\left.\left(\frac{\partial P}{\partial u}+\frac{\partial Q}{\partial v}\right)\right|_{\substack{u=u(x, y) \\
v=v(x, y)}} .
\end{aligned}
$$

By Lemma $13, M(h)$ can be analytically continued to $h=0$, with

$$
M(0)=0, \quad M^{\prime}(0)=\left.\frac{2 \pi}{\beta}\left(\frac{\partial P}{\partial u}+\frac{\partial Q}{\partial v}\right)\right|_{(u, v)=(0,0)} .
$$


Statement (1) of Theorem 3 follows from Proposition 10 and Lemma 15. Next, we prove statement (2) of this theorem.

Lemma 16. Let $H, \gamma_{h}, \omega$ be defined as in Lemma 15, then $M(h)=\int_{\gamma_{h}} \omega \equiv$ 0 if and only if there exists a real analytical function $z=z(u, v)$ defined in some neighbourhood of the origin $(0,0)$ satisfying the following linear partial differential equation:

$$
\frac{\partial H}{\partial u} \frac{\partial z}{\partial v}-\frac{\partial H}{\partial v} \frac{\partial z}{\partial u}=\frac{\partial P}{\partial u}+\frac{\partial Q}{\partial v}
$$

Proof. Sufficiency. Assume $z(u, v)$ satisfies Equation (14), then $\omega-z d H$ is a total differential of some function, i.e., there exists an analytical function $R$ defined in some neighbourhood of the origin such that $\omega-z d H=d R$. Therefore, $\int_{\gamma_{h}} \omega=\int_{\gamma_{h}}(z d H+d R) \equiv 0$.

Necessity. Let $u=u(x, y), v=v(x, y)$ be the area-preserving normalization transformation (13). We denote by

$$
\begin{aligned}
\bar{z}(x, y) & =z(u(x, y), v(x, y)), \\
\bar{H}(x, y) & =f\left(x^{2}+y^{2}\right)=H(u(x, y), v(x, y)), \\
F(x, y) & =\left.\left(\frac{\partial P}{\partial u}+\frac{\partial Q}{\partial v}\right)\right|_{\substack{u=u(x, y) \\
v=v(x, y)}} .
\end{aligned}
$$

Then

$$
\frac{\partial \bar{z}}{\partial x}=\frac{\partial z}{\partial u} \frac{\partial u}{\partial x}+\frac{\partial z}{\partial v} \frac{\partial v}{\partial x}, \quad \frac{\partial \bar{z}}{\partial y}=\frac{\partial z}{\partial u} \frac{\partial u}{\partial y}+\frac{\partial z}{\partial v} \frac{\partial v}{\partial y} .
$$

Consequently

$$
\left(\begin{array}{l}
\frac{\partial z}{\partial u} \\
\frac{\partial z}{\partial v}
\end{array}\right)=\left(\begin{array}{rr}
\frac{\partial v}{\partial y} & -\frac{\partial v}{\partial x} \\
-\frac{\partial u}{\partial y} & \frac{\partial u}{\partial x}
\end{array}\right)\left(\begin{array}{l}
\frac{\partial \bar{z}}{\partial x} \\
\frac{\partial \bar{z}}{\partial y}
\end{array}\right)
$$

Substituting it into (14), we get

$$
\left(\frac{\partial u}{\partial x} \frac{\partial \bar{z}}{\partial y}-\frac{\partial u}{\partial y} \frac{\partial \bar{z}}{\partial x}\right) \frac{\partial H}{\partial u}+\left(\frac{\partial v}{\partial x} \frac{\partial \bar{z}}{\partial y}-\frac{\partial v}{\partial y} \frac{\partial \bar{z}}{\partial x}\right) \frac{\partial H}{\partial v}=F(x, y)
$$

or equivalently

$$
\frac{\partial \bar{H}}{\partial x} \frac{\partial \bar{z}}{\partial y}-\frac{\partial \bar{H}}{\partial y} \frac{\partial \bar{z}}{\partial x}=F(x, y)
$$

By using $\bar{H}(x, y)=f\left(x^{2}+y^{2}\right),(15)$ can be written in the form

$$
\frac{\partial \bar{z}}{\partial y} x-\frac{\partial \bar{z}}{\partial x} y=R(x, y), \quad R(x, y):=\frac{F(x, y)}{2 f^{\prime}\left(x^{2}+y^{2}\right)} .
$$


If

$$
R(x, y)=\sum_{n=0}^{\infty} R_{n}(x, y), R_{n}(x, y)=\sum_{i=0}^{n} b_{i, n} x^{n-i} y^{i},
$$

then, by Lemma 13 and Remark 14,

$$
\int_{0}^{2 \pi} R(r \cos \theta, r \sin \theta) d \theta=\frac{1}{2 f^{\prime}\left(r^{2}\right)} \int_{0}^{2 \pi} F(r \cos \theta, r \sin \theta) d \theta \equiv 0 .
$$

This implies that the coefficients $b_{i, n}$ must satisfy (9). Let

$$
\bar{z}=\sum_{n=0}^{\infty} z_{n}, \quad z_{n}=\sum_{k=0}^{n} a_{k, n} x^{n-k} y^{k} .
$$

Substituting (17) into (16), we get

$$
\frac{\partial z_{n}}{\partial y} x-\frac{\partial z_{n}}{\partial x} y=R_{n}, \quad n=0,1,2, \ldots
$$

Setting $a_{-1, n}=a_{n+1, n}=0$, from (18), we obtain

$$
\sum_{k=0}^{n}\left[(k+1) a_{k+1, n}-(n-k+1) a_{k-1, n}\right] x^{n-k} y^{k}=\sum_{k=0}^{n} b_{k, n} x^{n-k} y^{k},
$$

or

$$
(k+1) a_{k+1, n}-(n-k+1) a_{k-1, n}=b_{k, n}, \quad k=0,1, \ldots, n .
$$

The determinant of system (19) is

$$
\begin{aligned}
\Delta & =\left|\begin{array}{ccccccc}
0 & 1 & 0 & \cdots & 0 & 0 & 0 \\
-n & 0 & 2 & \cdots & 0 & 0 & 0 \\
0 & 1-n & 0 & \cdots & 0 & 0 & 0 \\
\vdots & \vdots & \vdots & \ddots & \vdots & \vdots & \vdots \\
0 & 0 & 0 & \cdots & 0 & n-1 & 0 \\
0 & 0 & 0 & \cdots & -2 & 0 & n \\
0 & 0 & 0 & \cdots & 0 & -1 & 0
\end{array}\right| \\
& =\left\{\begin{array}{lll}
0 & & \text { if } n=2 m ; \\
{[(2 m+1) ! !]^{2}} & \text { if } n=2 m+1 .
\end{array}\right.
\end{aligned}
$$

Therefore, system (19) has a unique solution for $n$ odd. For $n=2 m$ even, system (19) can be divided into two independent systems:

$$
2 k a_{2 k, 2 m}-2(m-k+1) a_{2 k-2,2 m}=b_{2 k-1,2 m}, \quad k=1,2, \ldots, m,
$$

$$
(2 k+1) a_{2 k+1,2 m}-(2 m-2 k+1) a_{2 k-1,2 m}=b_{2 k, 2 m}, \quad k=0,1, \ldots, m .
$$

System (20) contains $m$ equations and $m+1$ unknown numbers and its matrix of coefficients has rank $m$. This implies that it has a solution of one 
dimension. System (21) contains $m+1$ equations and $m$ unknown numbers, and its matrix of coefficients has rank $m$. Note that the determinant of the augmented matrix of system (21) is

$$
\begin{aligned}
& \left|\begin{array}{ccccccc}
1 & 0 & 0 & \cdots & 0 & 0 & b_{0,2 m} \\
1-2 m & 3 & 0 & \cdots & 0 & 0 & b_{2,2 m} \\
0 & 3-2 m & 5 & \cdots & 0 & 0 & b_{4,2 m} \\
\vdots & \vdots & \vdots & \ddots & \vdots & \vdots & \vdots \\
0 & 0 & 0 & \cdots & 2 m-3 & 0 & b_{2 m-4,2 m} \\
0 & 0 & 0 & \cdots & -3 & 2 m-1 & b_{2 m-2,2 m} \\
0 & 0 & 0 & \cdots & 0 & -1 & b_{2 m, 2 m}
\end{array}\right| \\
= & \sum_{k=0}^{m}(2 m-2 k-1) ! !(2 k-1) ! ! b_{2 k, 2 m} \\
= & 0,
\end{aligned}
$$

the last equality follows from (9). Therefore, system (21) has a unique solution. The argument above shows that system (19) has always solutions, and if we set $a_{0,2 m}=0$, the solution is unique. Next we prove that the series (17) defined by the unique solution is convergent in some neighbourhood of the origin $(0,0)$. Assume that $R(x, y)$ is convergent in the square $D=$ $\left\{(x, y) \in \mathbf{C}^{2}|| x|\leq \bar{r}| y \mid, \leq \bar{r}\right\}$. Let $C=\sup _{D}|R|$, then by Cauchy inequality,

$$
\left|b_{i, n}\right| \leq \bar{r}^{-n} C \text {. }
$$

We claim that

$$
\left|a_{i, n}\right| \leq 2^{n} \bar{r}^{-n} C
$$

We will prove (23) only for $n=2 m+1, i=2 k+1$. All other cases can be proved in a similar way. Indeed, by (19),

$$
\begin{array}{r}
a_{-1, n}=0, \quad a_{1, n}=b_{0, n}, \quad a_{3, n}=\frac{1}{3} b_{2, n}+\frac{n-1}{3} b_{0, n}, \\
a_{5, n}=\frac{1}{5} b_{4, n}+\frac{n-3}{3 \cdot 5} b_{2, n}+\frac{(n-1)(n-3)}{3 \cdot 5} b_{0, n},
\end{array}
$$

and in general,

$$
a_{2 k+1, n}=\frac{1}{2 k+1} b_{2 k, n}+\frac{n-2 k+1}{2 k+1} a_{2 k-1, n} .
$$

Let

$$
e_{n}=\max _{k}\left\{\frac{(n-1)(n-3) \cdots(n-2 k+1)}{(2 k+1) ! !}\right\} \text {. }
$$


By induction,

$$
a_{2 k+1, n}=\sum_{j=0}^{k} l_{j, n} b_{2 j, n},
$$

where $l_{j, n}$ are some constants with $\left|l_{j, n}\right| \leq e_{n}$. Now we calculate the value of $e_{n}$. For $m=2 p$ even,

$$
\begin{aligned}
e_{n} & =\max _{k}\left\{\frac{4 p(4 p-2) \cdots(4 p-2 k+2)}{(2 k+1) ! !}\right\}=\frac{4 p(4 p-2) \cdots(2 p+2)}{(2 p+1) ! !} \\
& =\frac{(4 p) ! !}{(2 p+1) !}=\frac{2^{m}}{m+1} .
\end{aligned}
$$

Similarly, for $m=2 p+1$ odd, we also have $e_{n}=\frac{2^{m}}{m+1}$. Now from (24),

$$
\begin{aligned}
\left|a_{2 k+1, n}\right| & \leq \sum_{j=0}^{k} e_{n}\left|b_{2 j, n}\right| \leq 2^{m} \max _{j}\left|b_{2 j, n}\right| \\
& \leq 2^{m} \bar{r}^{-n} C \leq\left(\frac{2}{\bar{r}}\right)^{n} C
\end{aligned}
$$

which implies that (17) is convergent in the square $\left\{|x|<\frac{\bar{r}}{2},|y|<\frac{\bar{r}}{2}\right\}$.

Next we prove statement (2) of Theorem 3 by induction with respect to $k$.

Suppose $k=1$. The 1 -form $\omega_{1}-g_{1} d H$ with $g_{1}=z(x, y)$ is a total differential of some function if and only if $z(x, y)$ is a solution of (14). By Lemma 16 , if $\int_{\gamma_{h}} \omega_{1} \equiv 0$, then there exists an analytical function $g_{1}=z(x, y)$ defined in some neighbourhood of the origin satisfying (14). This implies that $\omega_{1}-g_{1} d H=d R_{1}$ for some analytical function $R_{1}$ defined in some neighbourhood of the origin. Therefore, $g_{1} \omega_{1}-\omega_{2}$ is an analytical 1-form defined in some neighbourhood of the origin. By Lemma 15 and Proposition $10, M_{2}(h)=\int_{\gamma_{h}} g_{1} \omega_{1}-\omega_{2}$ can be analytically continued to $h=0$ and $M_{2}(0)=0$. Now we assume that

$$
M_{j}(h)=\int_{\gamma_{h}}\left(\sum_{i=1}^{j-1} g_{i} \omega_{j-i}-\omega_{j}\right) \equiv 0 \text { for } 1 \leq j \leq k-1 .
$$

Applying Lemma 16 to the function $M_{k-1}(h)$, we get an analytical function $g_{k-1}$ defined in some neighbourhood of the origin $(0,0)$ such that $(6)$ holds for $i=k-1$. By Lemma 15 and Proposition 10, $M_{k}(h)=\int_{\gamma_{h}}\left(\sum_{i=1}^{k-1} g_{i} \omega_{k-i}-\right.$ $\left.\omega_{k}\right)$ can be analytically continued to $h=0$ and $M_{k}(0)=0$. Therefore, the proof of Theorem 3 is now completed. 


\section{Melnikov functions near homoclinic and heteroclinic cycles.}

In this section we shall prove Theorem 4 .

Lemma 17. Assume that $f \in C^{\omega}(\mathbf{R}, 0)$ with $f(0)=0, f^{\prime}(0)>0 ; P(u, v)$ and $Q(u, v)$ are analytical functions in the square $\left\{(u, v) \in \mathbf{C}^{2}|| u \mid \leq \delta_{1}\right.$, $\left.|v| \leq \delta_{1}\right\}$ :

$$
P(u, v)=\sum_{n=0}^{\infty} \sum_{i=0}^{n} p_{i, n} u^{n-i} v^{i}, \quad Q(u, v)=\sum_{n=0}^{\infty} \sum_{i=0}^{n} q_{i, n} u^{n-i} v^{i} .
$$

Let

$$
\begin{aligned}
D & =[-\delta, \delta] \times[-\delta, \delta] \subset \mathbf{R}^{2}, \quad 0<\delta<\delta_{1}, \\
\gamma_{h} & =\{(u, v) \in D \mid f(u v)=h, u \geq 0, v \geq 0\} .
\end{aligned}
$$

Define the function

$$
M(h)=\int_{\gamma_{h}} \omega, \quad \omega=-P d v+Q d u .
$$

Let $s=f^{-1}(h)$. Then there exist functions $a(s), b(s) \in C^{\omega}(\mathbf{R}, 0)$ such that

$$
M(h)=a(s)+b(s) \ln s, 0<h \ll 1,
$$

where

$$
a(0)=\int_{\gamma_{0}} \omega, b(s)=-\sum_{m=0}^{\infty}\left(p_{m, 2 m+1}+q_{m+1,2 m+1}\right) s^{m+1} .
$$

Proof. Calculating straightforwardly, we have

$$
\begin{aligned}
\int_{\gamma_{h}} Q d u= & \sum_{n=0}^{\infty} \sum_{i=0}^{n} q_{i, n} \int_{s \delta^{-1}}^{\delta} u^{n-2 i} s^{i} d u \\
= & \sum_{m=0}^{\infty} \sum_{i=0}^{2 m} q_{i, 2 m} \int_{s \delta^{-1}}^{\delta} u^{2 m-2 i} s^{i} d u \\
& +\sum_{m=0}^{\infty} \sum_{i=0}^{2 m+1} q_{i, 2 m+1} \int_{s \delta^{-1}}^{\delta} u^{2 m+1-2 i} s^{i} d u \\
= & \sum_{m=0}^{\infty} \sum_{i=0}^{2 m} \frac{q_{i, 2 m}}{2 m-2 i+1}\left(\delta^{2 m-2 i+1} s^{i}-\delta^{-2 m+2 i-1} s^{2 m-i+1}\right) \\
& +\sum_{m=0}^{\infty} \sum_{i=0}^{2 m+1} \frac{q_{i, 2 m+1}}{2 m-2 i+2}\left(\delta^{2 m-2 i+2} s^{i}-\delta^{-2 m+2 i-2} s^{2 m-i+2}\right) \\
& +2 \ln \delta \sum_{m=0}^{\infty} q_{m+1,2 m+1} s^{m+1}-\sum_{m=0}^{\infty} q_{m+1,2 m+1} s^{m+1} \ln s
\end{aligned}
$$




$$
=I_{1}(s)-\left(\sum_{m=0}^{\infty} q_{m+1,2 m+1} s^{m+1}\right) \ln s .
$$

Similarly, we can get

$$
\int_{\gamma_{h}} P d v=I_{2}(s)+\left(\sum_{m=0}^{\infty} p_{m, 2 m+1} s^{m+1}\right) \ln s,
$$

which, together with (25), implies

$$
M(h)=\int_{\gamma_{h}}-P d v+Q d u=a(s)+b(s) \ln s,
$$

where

$$
a(s)=I_{1}(s)-I_{2}(s), \quad b(s)=-\sum_{m=0}^{\infty}\left(p_{m, 2 m+1}+q_{m+1,2 m+1}\right) s^{m+1} .
$$

By using the Cauchy inequality, it is easy to prove that the functions $a(s)$ and $b(s)$ are analytical in some neighbourhood of $s=0$.

From Lemma 17 it follows:

Corollary 18. Under the assumption of Lemma 17, the function $M(h)$ can be analytically continued to $h=0$ if and only if

$$
p_{m, 2 m+1}+q_{m+1,2 m+1}=0, \quad \forall m \geq 0 .
$$

We remark that Condition (27) is equivalent to say that $b(s) \equiv 0$ for $0<s \ll 1$.

Lemma 19. Let $\gamma_{0}$ be as in Theorem 4 a heteroclinic cycle of system (3) consisting of $n$ hyperbolic saddles $p_{1}, p_{2}, \ldots, p_{n}$ (eventually they can be repeated) and $n$ separatrices, $\omega=-P(x, y) d y+Q(x, y) d x$ an analytical 1 -form defined in some neighbourhood of $\gamma_{0}$, and let $M(h):=\int_{\gamma_{h}} \omega$, then there exist analytical functions $a(h), b(h) \in C^{\omega}(\mathbf{R}, 0)$ with

$$
a(0)=\int_{\gamma_{0}} \omega, b(0)=0, b^{\prime}(0)=-\left.\sum_{i=1}^{n} \frac{1}{\lambda_{i}}\left(\frac{\partial P}{\partial x}+\frac{\partial Q}{\partial y}\right)\right|_{(x, y)=p_{i}}
$$

where $-\lambda_{i}<0<\lambda_{i}$ are the eigenvalues of the saddle $p_{i}$, such that

$$
M(h)=a(h)+b(h) \ln h, \quad 0<h \ll 1 .
$$

Moreover, if

$$
\left.\left(\frac{\partial P}{\partial x}+\frac{\partial Q}{\partial y}\right)\right|_{(x, y)=p_{i}}=0 \text { for } i=1,2, \ldots, n
$$

then

$$
a^{\prime}(0)=\int_{\gamma_{0}}\left(\frac{\partial P}{\partial x}+\frac{\partial Q}{\partial y}\right) d t
$$


Proof. According to Proposition 12, for every $1 \leq i \leq n$, there exist an analytical function $H_{i} \in C^{\omega}(\mathbf{R}, 0)$ with $H_{i}(0)=0, H_{i}^{\prime}(0)=\lambda_{i}$, and an area-preserving normalization coordinate transformation

$$
F_{i}: x=x(u, v, i), y=y(u, v, i)
$$

from some neighbourhood of the origin $(0,0)$ to some neighbourhood $U_{i}$ of $p_{i}$ such that in the new coordinate $(u, v)$ system (3) for $\epsilon=0$ takes the form

$$
\dot{u}=\frac{\partial G_{i}}{\partial v}, \quad \dot{v}=-\frac{\partial G_{i}}{\partial u}
$$

where

$$
G_{i}(u, v)=H_{i}(u v)=H \circ F_{i}(u, v) .
$$

Denote by $D=\{|u| \leq \delta,|v| \leq \delta\}$, and fix $\delta>0$ small enough such that $F_{i}(D) \subset U_{i}, i=1,2, \ldots, n$. We note that $u=0$ and $v=0$ are the separatrices of the saddle $(0,0)$ for the system $(\dot{u}, \dot{v})$. Let

$$
\Gamma_{i}^{+}=F_{i}(\{u=\delta\}), \quad \Gamma_{i}^{-}=F_{i}(\{v=\delta\}) .
$$

Any closed orbits near $\gamma_{0}$ is separated by $\Gamma_{i}^{ \pm}, i=1,2, \ldots, n$ into $2 n$ segments: $\gamma_{h}^{i}, i=1,2, \ldots, 2 n$, in which $\gamma_{h}^{2 i}$ are close to $p_{i}$ and $\gamma_{h}^{2 i-1}$ connects $\gamma_{h} \cap \Gamma_{i-1}^{+}$and $\gamma_{h} \cap \Gamma_{i}^{-}$, see Figure 2. Then

$$
M(h)=\int_{\gamma_{h}} \omega=\sum_{i=1}^{2 n} \int_{\gamma_{h}^{i}} \omega=\sum_{i=1}^{n} \int_{\gamma_{h}^{2 i}} \omega+\sum_{i=1}^{n} \int_{\gamma_{h}^{2 i-1}} \omega .
$$

Since $\gamma_{h}^{2 i-1}$ depend analytically on $h, \int_{\gamma_{h}^{2 i-1}} \omega$ are analytical at $h=0$. Next we consider the integrals $\int_{\gamma_{h}^{2 i}} \omega$. Let $\bar{\gamma}_{h}^{i}=\left\{(u, v) \in D \mid H_{i}(u v)=h\right\}$. Substituting $x=x(u, v, i), y=y(u, v, i)$ into the integral $\int_{\gamma_{h}^{2 i}} \omega$, we obtain

$$
\int_{\gamma_{h}^{2 i}} \omega=\int_{\bar{\gamma}_{h}^{i}}-\bar{P}_{i}(u, v) d v+\bar{Q}_{i}(u, v) d u
$$

where

$$
\bar{P}_{i}(u, v)=P \circ F_{i} \frac{\partial y}{\partial v}-Q \circ F_{i} \frac{\partial x}{\partial v}, \quad \bar{Q}_{i}(u, v)=Q \circ F_{i} \frac{\partial x}{\partial u}-P \circ F_{i} \frac{\partial y}{\partial u} .
$$




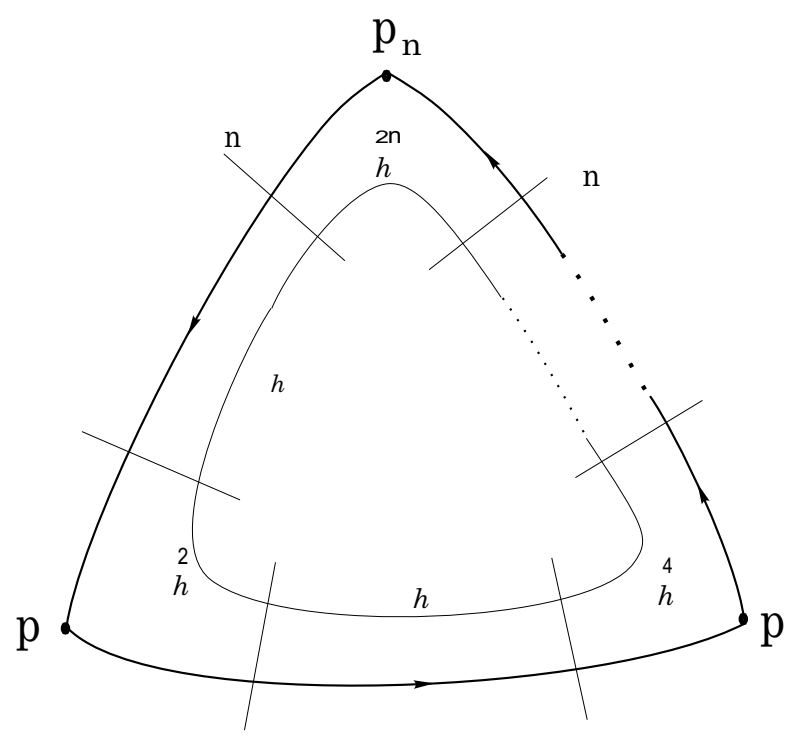

Figure 2.

Computing straightforward, we get

$$
\begin{aligned}
\frac{\partial \bar{P}_{i}}{\partial u}= & \left(\frac{\partial P}{\partial x} \frac{\partial x}{\partial u}+\frac{\partial P}{\partial y} \frac{\partial y}{\partial u}\right) \frac{\partial y}{\partial v}+P \circ F_{i} \frac{\partial^{2} y}{\partial u \partial v} \\
& -\left(\frac{\partial Q}{\partial x} \frac{\partial x}{\partial u}+\frac{\partial Q}{\partial y} \frac{\partial y}{\partial u}\right) \frac{\partial x}{\partial v}-Q \circ F_{i} \frac{\partial^{2} x}{\partial u \partial v}, \\
\frac{\partial \bar{Q}_{i}}{\partial u}= & \left(\frac{\partial Q}{\partial x} \frac{\partial x}{\partial v}+\frac{\partial Q}{\partial y} \frac{\partial y}{\partial v}\right) \frac{\partial x}{\partial u}+Q \circ F_{i} \frac{\partial^{2} x}{\partial u \partial v} \\
& -\left(\frac{\partial P}{\partial x} \frac{\partial x}{\partial v}+\frac{\partial P}{\partial y} \frac{\partial y}{\partial v}\right) \frac{\partial y}{\partial u}-P \circ F_{i} \frac{\partial^{2} y}{\partial u \partial v},
\end{aligned}
$$

which implies

$$
\begin{aligned}
\frac{\partial \bar{P}_{i}}{\partial u}+\frac{\partial \bar{Q}_{i}}{\partial v} & =\frac{\partial P}{\partial x}\left(\frac{\partial x}{\partial u} \frac{\partial y}{\partial v}-\frac{\partial x}{\partial v} \frac{\partial y}{\partial u}\right)+\frac{\partial Q}{\partial y}\left(\frac{\partial x}{\partial u} \frac{\partial y}{\partial v}-\frac{\partial x}{\partial v} \frac{\partial y}{\partial u}\right) \\
& =\left(\frac{\partial P}{\partial x}+\frac{\partial Q}{\partial y}\right) \circ F_{i}
\end{aligned}
$$

The last equality above follows from the fact that $F_{i}$ is area-preserving. Let $s=H_{i}^{-1}(h)$. From Lemma 17, there exist analytical functions $a_{i}(s)$, $b_{i}(s) \in C^{\omega}(\mathbf{R}, 0)$ with $a_{i}(0)=\int_{\bar{\gamma}_{0}^{i}}-\bar{P}_{i} d v+\bar{Q}_{i} d u, b_{i}(0)=0$ and $b_{i}^{\prime}(0)=$ 
$\left.\left(\frac{\partial \bar{P}_{i}}{\partial u}+\frac{\partial \bar{Q}_{i}}{\partial y}\right)\right|_{(u, v)=(0,0)}$ such that

$$
\int_{\gamma_{h}^{2 i}} \omega=\int_{\bar{\gamma}_{h}^{i}}-\bar{P}_{i} d v+\bar{Q}_{i} d u=a_{i}(s)+b_{i}(s) \ln s, \quad 0<h \ll 1 .
$$

Consequently,

$$
\int_{\gamma_{h}^{2 i}} \omega=\bar{a}_{i}(h)+\bar{b}_{i}(h) \ln h,
$$

where

$$
\bar{a}_{i}(h)=a_{i} \circ H_{i}^{-1}(h)+b_{i} \circ H_{i}^{-1}(h) \ln \left(\frac{H_{i}^{-1}(h)}{h}\right) \text { and } \bar{b}_{i}(h)=b_{i} \circ H_{i}^{-1}(h)
$$

are analytical at $h=0$ and satisfy

$$
\begin{aligned}
\bar{b}_{i}(0) & =b_{i}(0)=0 \\
\bar{b}_{i}^{\prime}(0) & =\frac{b_{i}^{\prime}(0)}{\lambda_{i}}=-\left.\frac{1}{\lambda_{i}}\left(\frac{\partial \bar{P}}{\partial u}+\frac{\partial \bar{Q}}{\partial v}\right)\right|_{(u, v)=(0,0)} \\
& =-\left.\frac{1}{\lambda_{i}}\left(\frac{\partial P}{\partial x}+\frac{\partial Q}{\partial y}\right)\right|_{(x, y)=p_{i}} .
\end{aligned}
$$

Substituting (32) into (30), we obtain

$$
M(h)=a(h)+b(h) \ln h,
$$

where

$$
a(h)=\sum_{i=1}^{n} \int_{\gamma_{h}^{2 i-1}} \omega+\sum_{i=1}^{n} \bar{a}_{i}(h), \quad b(h)=\sum_{i=1}^{n} \bar{b}_{i}(h)
$$

are analytical at $h=0$ and satisfy

$$
\begin{aligned}
a(0) & =\sum_{i=1}^{n} \int_{\gamma_{0}^{2 i-1}} \omega+\sum_{i=1}^{n} \bar{a}_{i}(0)=\sum_{i=1}^{n} \int_{\gamma_{0}^{2 i-1}} \omega+\sum_{i=1}^{n} \int_{\gamma_{0}^{2 i}} \omega=\int_{\gamma_{0}} \omega, \\
b(0) & =\sum_{i=1}^{n} \bar{b}_{i}(0)=0 \\
b^{\prime}(0) & =\sum_{i=1}^{n} \bar{b}_{i}^{\prime}(0)=-\left.\sum_{i=1}^{n} \frac{1}{\lambda_{i}}\left(\frac{\partial P}{\partial x}+\frac{\partial Q}{\partial y}\right)\right|_{(x, y)=p_{i}} .
\end{aligned}
$$

Now we prove (29). First we point out that if (28) holds, it follows from $b(0)=b^{\prime}(0)=0$ that $M(h) \in C^{1}$. We claim that the integral in (29) is convergent. Indeed, let $p(t) \subset \gamma_{0}$ be a solution of system (3) for $\epsilon=0$ and assume that $\lim _{t \rightarrow+\infty} p(t)=p_{i}, \lim _{t \rightarrow-\infty} p(t)=p_{i-1}$. Note that $p_{i}$ is a 
hyperbolic saddle, we have, as $t \rightarrow+\infty,\left\|p(t)-p_{i}\right\|=O(\exp (-c t))$ for some $c>0$. Hence,

$$
\begin{aligned}
\left\|\left(\frac{\partial P}{\partial x}+\frac{\partial Q}{\partial y}\right) \circ p(t)\right\| & =\left\|\left(\frac{\partial P}{\partial x}+\frac{\partial Q}{\partial y}\right) \circ p(t)-\left.\left(\frac{\partial P}{\partial x}+\frac{\partial Q}{\partial y}\right)\right|_{p_{i}}\right\| \\
& =O\left(\left\|p(t)-p_{i}\right\|\right)=O(\exp (-c t)), \text { as } t \rightarrow+\infty
\end{aligned}
$$

So, the integral $\int_{0}^{+\infty}\left(\frac{\partial P}{\partial x}+\frac{\partial Q}{\partial y}\right) d t$ is convergent. Similarly, the integral $\int_{-\infty}^{0}\left(\frac{\partial P}{\partial x}+\frac{\partial Q}{\partial y}\right) d t$ is convergent too. Our claim is proved. From $H(x, y)=$ $h$, we get

$$
\frac{\partial H}{\partial x} \frac{\partial x}{\partial h}=1, \quad \frac{\partial H}{\partial y} \frac{\partial y}{\partial h}=1
$$

which implies

$$
\frac{\partial x}{\partial h} d y=-d t, \quad \frac{\partial y}{\partial h} d x=d t
$$

Thus,

$$
\begin{aligned}
M^{\prime}(h) & =\frac{\partial}{\partial h} \int_{\gamma_{h}}(-P(x, y) d y+Q(x, y) d x) \\
& =\int_{\gamma_{h}}\left(-\frac{\partial P}{\partial x} \frac{\partial x}{\partial h} d y+\frac{\partial Q}{\partial y} \frac{\partial y}{\partial h} d x\right)=\int_{\gamma_{h}}\left(\frac{\partial P}{\partial x}+\frac{\partial Q}{\partial y}\right) d t .
\end{aligned}
$$

Let $h \rightarrow 0$, we get

$$
a^{\prime}(0)=M^{\prime}(0)=\lim _{h \rightarrow 0} M^{\prime}(h)=\lim _{h \rightarrow 0} M^{\prime}(h)=\int_{\gamma_{0}}\left(\frac{\partial P}{\partial x}+\frac{\partial Q}{\partial y}\right) d t .
$$

From Lemma 19, we get immediately Statement (1) of Theorem 4. Next we prove Statement (2).

Lemma 20. Let $\omega=-P(x, y) d y+Q(x, y) d x$ be an analytical 1-form defined in some neighbourhood of $\gamma_{0}$, where $\gamma_{0}$ is a homoclinic orbit of a hyperbolic saddle $p=(0,0)$ of system $(7)$, then $M(h):=\int_{\gamma_{h}} \omega$ can be analytically continued to $h=0$ if and only if for any area-preserving normalization coordinate transformation $F(u, v)$ near $p$ given by Proposition 12, the Taylor series of the function $\left(\frac{\partial P}{\partial x}+\frac{\partial Q}{\partial y}\right) \circ F(u, v)$ at $(0,0)$ does not contain the terms $u^{m} v^{m}$ for any integers $m \geq 0$.

Proof. According to Proposition 12, there exists a function $f \in C^{\omega}(\mathbf{R}, 0)$ with $f(0)=0, f^{\prime}(0)>0$ such that $f(u v)=H \circ F(u, v)$. Let

$$
\Gamma^{+}=F(\{u=\delta\}), \quad \Gamma^{-}=F(\{v=\delta\}) .
$$


Any closed orbits near $\gamma_{0}$ is separated by $\Gamma^{ \pm}$into two segments: $\gamma_{h}^{1}$ and $\gamma_{h}^{2}$, in which $\gamma_{h}^{2}$ is close to the saddle $p$ and $\gamma_{h}^{1}$ connects $\gamma_{h} \cap \Gamma^{+}$and $\gamma_{h} \cap \Gamma^{-}$ in the complement of some neighbourhood of $p$. Since the integral $\int_{\gamma_{h}^{1}} \omega$ is analytical at $h=0$,

$$
M(h)=\int_{\gamma_{h}} \omega=\int_{\gamma_{h}^{1}} \omega+\int_{\gamma_{h}^{2}} \omega
$$

can be analytically continued to $h=0$ if and only if the integral $\int_{\gamma_{h}^{2}} \omega$ can be analytically continued. Let $\bar{\gamma}_{h}^{2}=\{(u, v) \mid f(u v)=h, 0<u, v \leq \delta\}$, then as in the proof of Lemma 19, we have

$$
\int_{\gamma_{h}^{2}} \omega=\int_{\bar{\gamma}_{h}^{2}}-\bar{P}(u, v) d v+\bar{Q}(u, v) d u
$$

where

$$
\bar{P}(u, v)=P \circ F \frac{\partial y}{\partial v}-Q \circ F \frac{\partial x}{\partial v}, \quad \bar{Q}(u, v)=Q \circ F \frac{\partial x}{\partial u}-P \circ F \frac{\partial y}{\partial u} .
$$

Let

$$
\bar{P}(u, v)=\sum_{n=0}^{\infty} \sum_{i=0}^{n} p_{i, n} u^{n-i} v^{i}, \quad \bar{Q}(u, v)=\sum_{n=0}^{\infty} \sum_{i=0}^{n} q_{i, n} u^{n-i} v^{i} .
$$

From Corollary 18, the integral $\int_{\gamma_{h}^{2}} \omega$ can be analytically continued to $h=0$ if and only if (27) holds which is equivalent to say that the coefficients of the terms $u^{m} v^{m}$ for any $m \geq 0$ in the Taylor series of the function $\frac{\partial \bar{P}}{\partial u}+\frac{\partial \bar{Q}}{\partial v}$ at $(0,0)$ are zero. Now the statement of Lemma 20 follows from $(31)$.

Lemma 21. Let $\omega$ and $M(h)$ be defined as in Lemma 20, then the function $M(h)$ can be analytically continued to $h=0$ if and only if there exists an analytical function $z=z(x, y)$ defined in some neighbourhood of the saddle $p$, such that $z(x, y)$ satisfies the following linear partial differential equation:

$$
\frac{\partial z}{\partial x} \frac{\partial H}{\partial y}-\frac{\partial z}{\partial y} \frac{\partial H}{\partial x}=\frac{\partial P}{\partial x}+\frac{\partial Q}{\partial y} .
$$

Proof. Let $(x, y)=F(u, v)$ be the area-preserving normalization coordinate transformation near $p$ given by Proposition 12 and let $\bar{z}=z \circ F, f(u v)=$ $H \circ F(u, v)$, then (34) can be changed to the form

$$
\frac{\partial \bar{z}}{\partial u} u-\frac{\partial \bar{z}}{\partial v} v=R(u, v), R(u, v)=\frac{1}{f^{\prime}(u v)}\left(\frac{\partial P}{\partial x}+\frac{\partial Q}{\partial y}\right) \circ F(u, v) .
$$

Obviously, the Taylor series of $f^{\prime}(u v) R(u, v)$ at $(0,0)$ does not contain the terms $u^{m} v^{m}$ for any integers $m \geq 0$ if and only if $R(u, v)$ has the same 
property. Let

$$
\begin{aligned}
& R(u, v)=\sum_{n=0}^{\infty} \sum_{i=0}^{n} b_{i, n} u^{n-i} v^{i}, \\
& \bar{z}(u, v)=\sum_{n=0}^{\infty} \sum_{i=0}^{n} a_{i, n} u^{n-i} v^{i} .
\end{aligned}
$$

Substituting them into (35), we get

$$
\sum_{n=0}^{\infty} \sum_{i=0}^{n}\left[(n-i) a_{i, n}-a_{i, n} i\right] u^{n-i} v^{i}=\sum_{n=0}^{\infty} \sum_{i=0}^{n} b_{i, n} u^{n-i} v^{i},
$$

or equivalently

$$
(n-2 i) a_{i, n}=b_{i, n}, \quad \text { for } n=0,1,2, \ldots, \text { and } i=0,1, \ldots, n \text {. }
$$

System (38) has solutions if and only if

$$
b_{m, 2 m}=0, \forall m \geq 0 .
$$

Moreover, if (39) holds, we can choose

$$
a_{i, n}=\left\{\begin{array}{cl}
\frac{b_{i, n}}{n-2 i}, & \text { if } n \neq 2 i \\
0, & \text { if } n=2 i
\end{array}\right.
$$

Since $\left|a_{i, n}\right| \leq\left|b_{i, n}\right|$, the convergence radius of (37) is at least equal to the convergence radius of (36). So the function $\bar{z}$ defined in (37) is analytical in some neighbourhood of the origin. Now, the lemma follows using Lemma 20.

Lemma 22. Let $\omega$ and $M(h)$ be defined as in Lemma 20, then the function $M(h)$ is constant for $0<h \ll 1$ if and only if there exists an analytical function $z=z(x, y)$ defined in some neighbourhood of $\gamma_{0}$ such that (34) holds.

Proof. Sufficiency. We consider the characteristic equation of (34):

$$
\dot{x}=\frac{\partial H}{\partial y}, \quad \dot{y}=-\frac{\partial H}{\partial x}, \quad \dot{z}=\frac{\partial P}{\partial x}+\frac{\partial Q}{\partial y} .
$$

Assume that there exists an analytical function $z=z(x, y)$ defined in some neighbourhood of $\gamma_{0}$ such that $z(x, y)$ satisfies Equation (34). This implies that the surface $S=\left\{(x, y, z) \in \mathbf{R}^{3} \mid z=z(x, y)\right\}$ is invariant under the flow of (40). Therefore, $S \cap\left\{(x, y, z) \in \mathbf{R}^{3} \mid H(x, y)=h\right\}$ for $0<h \ll 1$ is a periodic orbit of (40). So from (33), we have

$$
M^{\prime}(h)=\int_{\gamma_{h}}\left(\frac{\partial P}{\partial x}+\frac{\partial Q}{\partial y}\right) d t=\int_{\gamma_{h}} \dot{z} d t=0,
$$

which implies that $M(h)$ is constant for $0<h \ll 1$. 
Necessity. Assume now $M(h)$ is constant. By Lemma 21, Equation (34) has an analytical solution $z(x, y)$ in some neighbourhood of the saddle $p=$ $(0,0)$. We claim that this solution $z(x, y)$ can be extended continuously to a single valued analytic function in some neighbourhood of $\gamma_{0}$. Indeed, from (34), $\left.\left(\frac{\partial P}{\partial x}+\frac{\partial Q}{\partial y}\right)\right|_{(x, y)=(0,0)}=0$, so the straight line $\{(0,0, z) \mid z \in$ $\mathbf{R}$ \} consists of singular points of (40). Then the invariant surface $S$ of (40) contains local stable and unstable manifolds of the singular point $p_{0}=$ $(0,0, z(0,0))$. Let $\Gamma^{-}$and $\Gamma^{+}$be the planes transversal to the flow of (40) at some point of local stable manifold and local unstable one respectively. Then $l^{ \pm}:=S \cap \Gamma^{ \pm}$are analytical curves in $\Gamma^{ \pm}$, see Figure 3 .

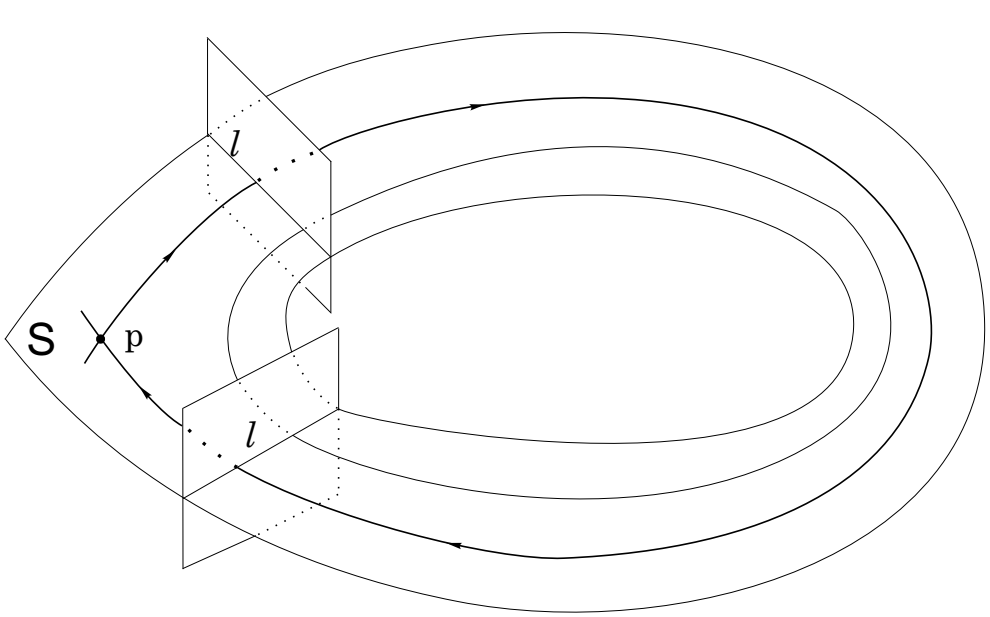

Figure 3.

Let $A$ be the projection from $\Gamma^{+}$to $\Gamma^{-}$along the orbits of (40). Then $l_{-}^{\prime}:=A l^{+}$is an analytical curve in $\Gamma^{-}$. Introducing the set $U=\{(x, y, z) \in$ $\left.\mathbf{R}^{3} \mid H(x, y)>0\right\}$, by (41) we have $U$ is filled with periodic orbits. This implies $l_{-}^{\prime} \cap U=l^{-} \cap U$. Therefore, by the analyticity, $l_{-}^{\prime}=l^{-}$. Thus, the union of the orbits passing through $l^{+}$and $S$ constructs an analytical invariant surface of system (40), which is the graph of an analytical function $z(x, y)$ defined in some neighbourhood of $\gamma_{0}$. From the invariance, $z(x, y)$ is a solution of Equation (34).

Lemma 23. Let $\omega$ and $M(h)$ be defined as in Lemma 20. Then $M(h) \equiv 0$ if and only if there exist analytical functions $z(x, y)$ and $R(x, y)$ defined in some neighbourhood of $\gamma_{0}$ such that

$$
\omega=z d H+d R .
$$


Proof. Sufficiency. Assume that formula (42) holds. Since the function $H$ is constant along the closed curve $\gamma_{h}$, so $d H=0$. This implies that $\int_{\gamma_{h}} \omega=\int_{\gamma_{h}} z d H+d R=\int_{\gamma_{h}} d R=0$.

Necessity. By Lemma 22, there exists an analytical function $z(x, y)$ defined in some neighbourhood of $\gamma_{0}$ such that Equation (34) holds. Let

$$
M d x+N d y=\omega-z d H .
$$

From (34),

$$
\frac{\partial M}{\partial y}=\frac{\partial N}{\partial x} .
$$

Now we define the function $R(x, y)$ in the following way: For any point $(x, y)$ near $\gamma_{0}$, let

$$
R(x, y)=\int_{(0,0)}^{(x, y)} M d x+N d y
$$

By (43) and the fact

$$
\int_{\gamma_{h}} M d x+N d y=\int_{\gamma_{h}}(\omega-z d H)=\int_{\gamma_{h}} \omega=M(h) \equiv 0,
$$

the integral in (44) defines a single valued analytical function in some neighbourhood of $\gamma_{0}$ satisfying (42).

The proof of statement (2) of Theorem 4. Suppose $k=2$. Then $M_{1}(h)=$ $-\int_{\gamma_{h}} \omega_{1} \equiv 0$. By Lemma 23, there exist analytical functions $z_{1}, R_{1}$ defined in some neighbourhood of $\gamma_{0}$ such that $\omega_{1}=z_{1} d H+d R_{1}$. By Proposition 10, $M_{2}(h)=\int_{\gamma_{h}}\left(z_{1} \omega_{1}-\omega_{2}\right)$. Now by using Lemma 19, we get that statement (2) holds for $k=2$. Similarly, assume that $M_{i}(h) \equiv 0,1 \leq i \leq k-1$. Again from Lemma 23, there exist analytical functions $a_{k-1}, R_{k-1}$ defined in some neighbourhood of $\gamma_{0}$ satisfying (6). Now by Proposition 10 and Lemma 19, Statement (2) holds for $k$. This completes the proof of Theorem 4.

Proof of Theorem 5. Statement (1) is just a corollary of Lemma 20. Statement (2) is a corollary of Lemma 24 below. Finally Statement (3) follows easily from Lemma 24 and Lemma 19.

All notations used in Lemma 24 below are the same as in the statement of Theorem 5.

Lemma 24. Let $\omega$ be an analytical 1-form defined in some neighbourhood of the eight figure cycle, then two of the three integrals $\int_{\gamma_{h}} \omega, \int_{\gamma_{h}^{+}} \omega, \int_{\gamma_{h}^{-}} \omega$ are identically zero if and only if there exist analytical functions $z(x, y), R(x, y)$ defined in some neighbourhood of $\gamma_{0}^{+} \cup \gamma_{0}^{-}$such that $\omega=z d H+d R$. 
Proof. The sufficiency can be proved by using the same argument as the proof of Lemma 23. We now prove the necessity. First by Lemma 21, Equation (34) has an analytical solution $z(x, y)$ in some neighbourhood of the saddle $p$. By using the same argument of the proof of Lemma 22, the function $z(x, y)$ can be extended continuously to a single valued analytic function in the some neighbourhood of the eight figure. Let $M d x+N d y=$ $\omega-z d H$. Then from (34), formula (43) holds. Now we define the function $R$ by the integral in (44). Then by (43) and the assumption that two of the three integrals $\int_{\gamma_{h}} \omega, \int_{\gamma_{h}^{+}} \omega, \int_{\gamma_{h}^{-}} \omega$ are identically zero, the function $R$ is a single-valued analytical one in some neighbourhood of eight figure $\gamma_{0}^{+} \cup \gamma_{0}^{-}$ and satisfies (42).

\section{Proof of Theorem 8.}

We shall need the following result:

Proposition 25. Let $X$ be an analytical vector field defined in some open region of $\mathbf{R}^{2}$. Assume that $X$ has a continuous family of periodic orbits (the period annulus) $\gamma_{s}, 0<s<\bar{s}$, and $\gamma_{0}$ is a nondegenerate center $p$ or a homoclinic orbit of a hyperbolic saddle $p$ such that $\lim _{s \backslash 0} \gamma_{s}=\gamma_{0}$, then for any $s \in[0, \bar{s})$, there exists an analytical function $\rho>0$ defined in some neighbourhood of $\gamma_{s}$ such that $\operatorname{div}(\rho X) \equiv 0$, i.e., $\rho X$ is a Hamiltonian vector field.

Proposition 25 will be proved through two lemmas.

Lemma 26. Under the assumptions of Proposition 25 the following hold: For any $s \in[0, \bar{s})$, the vector field $X$ has an analytical first integral $H$ defined in some neighbourhood of $\gamma_{s}$ such that

$$
\operatorname{det} D^{2} H(p) \neq 0 \text { and } D H=0 \Leftrightarrow X=0 .
$$

Proof. If $\gamma_{s}$ is a periodic orbit, the lemma is trivial. For $\gamma_{0}$ being a nondegenerate center, by the Poincaré Normal Form Theorem (for a proof, see $[\mathbf{1}])$, there exists an analytic change of coordinates that brings the initial system to the normal form

$$
\begin{aligned}
& \dot{x}=-y f\left(x^{2}+y^{2}\right), \\
& \dot{y}=x f\left(x^{2}+y^{2}\right) .
\end{aligned}
$$

Obviously, the system above has a first integral $H=x^{2}+y^{2}$ satisfying (45). Now we assume that $\gamma_{0}$ is a homoclinic orbit of a hyperbolic saddle. Since there exists a family of periodic orbits tending to $\gamma_{0}$, the saddle values of any order must be zero. Therefore under the normalized coordinate the vector field $X$ near the saddle takes the following form (see [1]):

$$
\begin{aligned}
& \dot{x}=-\lambda x(1+R(x y)) \\
& \dot{y}=\lambda y(1+R(x y)), R \in C^{\omega}(\mathbf{R}, 0), R(0)=0 .
\end{aligned}
$$


$H=x y$ is a first integral of system (46). This implies that the vector field $X$ has an analytical first integral in some neighbourhood $U$ of the saddle satisfying (45). We claim that the first integral $H$ can be extended to some neighbourhood of $\gamma_{0}$. Indeed, let $I, J \subset U$ be two transversal sections to $X$ at some point of the local stable manifold and the local unstable one, respectively. Sections $I$ and $J$ can be parameterized by using $h=H$. Without loss of generality, we assume that the intersections of the periodic orbits $\gamma_{s}$ with $I$ and $J$ correspond to points with $h>0$. Let $f: J \rightarrow I$ denote the Poincaré map along the orbits of $X$, then $f \in C^{\omega}(\mathbf{R}, 0)$. Let $G(h)=H(f(h))-h$. Since all orbits starting from the points of $J$ with $h>0$ are periodic, we have $G(h) \equiv 0$ for $0<h \ll 1$. By the analyticity, $G(h) \equiv 0$ for $|h| \ll 1$. Now we define the function $H$ in some neighbourhood of $\gamma_{0}$ as follows: For any $x$ close to $\gamma_{0}$, denote by $\gamma(x)$ the orbit of $X$ passing through $x$, then $H(x):=H(\gamma(x) \cap I)=H(\gamma(x) \cap J)$. Obviously, $H(x)$ takes a constant on each orbit, i.e., $H$ is a first integral.

Let $H$ be the first integral in some neighbourhood of $\gamma_{s}$ as in Lemma 26 . Consider the Hamiltonian vector field

$$
Y:=J D H, J=\left(\begin{array}{cc}
0 & 1 \\
-1 & 0
\end{array}\right)
$$

Then $X$ and $Y$ define the same direction field. Let $\rho=\|Y\| /\|X\|$, then $\rho$ is positive and analytical at any regular point. Obviously, $Y=\rho X$ or $Y=-\rho X$. The next lemma shows that the function $\rho$ can be analytically continued to the saddle $p$.

Lemma 27. Assume that the analytical vector fields $X_{1}=\left(P_{1}, Q_{1}\right)$ and $X_{2}=\left(P_{2}, Q_{2}\right)$ define the same direction field and the origin $(0,0)$ is an isolated singular point of $X_{1}$ and $X_{2}$. If

$$
\left.\prod_{i=1}^{2}\left[\left(\frac{\partial P_{i}}{\partial x}\right)^{2}+\left(\frac{\partial P_{i}}{\partial y}\right)^{2}\right]\right|_{(0,0)} \neq 0
$$

or

$$
\left.\prod_{i=1}^{2}\left[\left(\frac{\partial Q_{i}}{\partial x}\right)^{2}+\left(\frac{\partial Q_{i}}{\partial y}\right)^{2}\right]\right|_{(0,0)} \neq 0,
$$

then there exists a positive analytical function $\rho$ defined in some neighbourhood of the origin such that $\left\|X_{1}\right\|=\rho\left\|X_{2}\right\|$.

Proof. We will prove the lemma under the assumption $\frac{\partial P_{1}}{\partial x}(0,0) \neq 0$. The proofs for other cases are similar. Since $X_{1}$ and $X_{2}$ define the same direction field, they have the same vertical isocline:

$$
V:\left\{(x, y) \mid P_{1}(x, y)=0\right\}=\left\{(x, y) \mid P_{2}(x, y)=0\right\} .
$$


By using Implicit Function Theorem for $P_{1}$ at $(0,0)$, there exists a function $g(y) \in C^{\omega}(\mathbf{R}, 0)$ with $g(0)=0$, such that

$$
V=\{(x, y) \mid x=g(y)\} .
$$

Thus,

$$
P_{1}(x, y)=(x-g(y)) \bar{P}_{1}(x, y), \bar{P}_{1} \in C^{\omega}\left(\mathbf{R}^{2}, \mathbf{0}\right), \bar{P}_{1}(0,0)=\frac{\partial P_{1}}{\partial x}(0,0) \neq 0 .
$$

We claim that $\frac{\partial P_{2}}{\partial x}(0,0) \neq 0$. Indeed, if $\frac{\partial P_{2}}{\partial x}(0,0)=0$, then by $(47), \frac{\partial P_{2}}{\partial y}(0,0)$ $\neq 0$. By the Implicit Function Theorem, the curve $V$ is tangent to the $x$-axis at the origin, which is a contradiction with (48). Thus, we get

$$
P_{2}(x, y)=(x-g(y)) \bar{P}_{2}(x, y), \bar{P}_{2} \in C^{\omega}\left(\mathbf{R}^{2}, \mathbf{0}\right), \bar{P}_{2}(0,0)=\frac{\partial P_{2}}{\partial x}(0,0) \neq 0 .
$$

Therefore, the function $\bar{\rho}(x, y):=\frac{\bar{P}_{1}(x, y)}{\bar{P}_{2}(x, y)}$ is analytical and has definite sign in some neighbourhood of the origin. Let $\rho=|\bar{\rho}|$, then the proof of the lemma is completed.

Proof of Proposition 25. It is a corollary of Lemmas 26 and 27.

Proof of Theorem 8. Consider the following one parameter family of analytical systems:

$$
\dot{x}=f(x)+\epsilon g(x, \epsilon), \quad x \in \mathbf{R}^{2} .
$$

We assume that for $\epsilon=0$ system (49) has a homoclinic orbit $\gamma_{0}$ of a hyperbolic saddle of infinite codimension, i.e., there is a continuous family of periodic orbits tending to $\gamma_{0}$. By Proposition 25, there exists a positive analytical function $\rho(x)$ defined in some neighbourhood of $\gamma_{0}$ such that $\operatorname{div}(\rho f) \equiv 0$. Now we consider the following perturbed Hamiltonian system which is orbitally equivalent to system (49):

$$
\dot{x}=\rho(x) f(x)+\epsilon \rho(x) g(x, \epsilon) .
$$

We assume, without loss of generality, that there exists an analytical function $H$ defined in some neighbourhood of $\gamma_{0}$ such that $J D H=\rho f, \gamma_{0} \subset$ $H^{-1}(0)$, and for $0<h \ll 1, \gamma_{h} \subset H^{-1}(h)$ is a family of periodic orbits with $\gamma_{h} \rightarrow \gamma_{0}$ as $h \searrow 0$. Let $M_{k}(h)$ denote the $k$-th Melnikov function of system (50).

Case 1. $\quad M_{i}(h) \equiv 0, \forall i \geq 1$. Then for $|\epsilon| \ll 1$, system (50) has a period annulus and hence has no limit cycles near $\gamma_{0}$.

Case 2. There exists an integer $k \geq 1$ such that $M_{i}(h) \equiv 0,0 \leq i \leq k-1$ and $M_{k}(h)=a_{k}(h)+b_{k}(h) \ln h$ is not identically vanishing. Then by Remark 7 , system (50) in some neighbourhood of $\gamma_{0}$ has at most $2 \min \left\{m\left(a_{k}\right), m\left(b_{k}\right)\right\}$ limit cycles for $|\epsilon| \ll 1$.

This completes the Proof of Theorem 8. 
Acknowledgement. Weigu $\mathrm{Li}$ and Xiang Zhang want to thank the CRM and the Department of Mathematics of the Universitat Autònoma de Barcelona for their support and hospitality during the period in which this paper was written. They are partially supported by NSFC of China and Weigu Li is supported by the 973 Project of the Ministry of Science and Technology of China. Jaume Llibre is partially supported by DGES grant number PB96-1153 and by CIRIT grant number 1999SER-00349.

\section{References}

[1] Yu.N. Bibikov, Local Theory of Nonlinear Analytic Ordinary Differential Equations, Lect. Notes Math., 702, Springer Verlag, Berlin, Heidelberg, New York, 1979, MR 83a:34004, Zbl 0404.34005.

[2] I.S. Gradshteyn and I.M. Ryzhik, Table of Integrals, Series and Products, Academic Press, San Diego, 1980, MR 81g:33001, Zbl 0521.33001.

[3] L. Hörmander, An Introduction to Complex Analysis in Several Variables, NorthHolland mathematical library, 7, Amsterdam; New York, Oxford, Tokyo, 1990, MR 91a:32001, Zbl 0685.32001.

[4] I.D. Iliev, Higher order Melnikov functions for degenerate cubic Hamiltonians, Adv. Differential Equations, 1 (1996), 689-708, MR 97k:34039, Zbl 0851.34042.

[5] Yu. Il'yashenko and S. Yakovenko, Finitely-smooth normal forms for local families of diffeomorphisms and vector fields, Russian Math. Surveys, 46 (1991), 1-43, MR 92i:58165, Zbl 0744.58006.

[6] H. Ito, Convergence of Birkhoff normal forms for integrable systems, Comment. Math. Helv., 64 (1989), 412-461, MR 90f:34070, Zbl 0686.58021.

[7] Q. Jiang and M. Han, Melnikov functions and perturbation of planar Hamiltonian systems, Chinese Ann. of Math. Ser. B, 20 (1999), 233-246, MR 2000d:34055, Zbl 0931.34020.

[8] J. Moser, The analytic invariants of an area-preserving mapping near a hyperbolic fixed point, Comm. Pure Appl. Math., 9 (1956), 673-692, MR 19,278b, Zbl 0072.40801.

[9] J.C. Poggiale, Applications des Variétés Invariantes à la Modélisation de l'Hétérogénéité Endynamique des Populations, Thèse, Université de Bourgogne, 1994.

[10] R. Roussarie, On the number of limit cycles which appear by perturbation of separatrix loop of planar vector fields, Bol. Soc. Bras. Mat., 17 (1986), 67-101, MR 88i:34061, Zbl 0628.34032.

[11] _ Cyclicité finie des lacets et des points cuspidaux, Nonlinearity, 2 (1989), 73-117, MR 90m:58169, Zbl 0679.58037.

[12] _ Bifurcation of Planar Vector Fields and Hilbert's Sixteenth Problem, Progress in Mathematics, 164, Basel, Boston, Berlin, Birkhäuser, 1998, MR 99k:58129, Zbl 0898.58039. 
Received May 10, 2001 and revised May 2, 2002.

Department of Mathematics

Peking University,

BEIJING 100871

P.R. ChinA

E-mail address: weigu@math.pku.edu.cn

Departament de Matemàtiques

Universitat Autònoma de Barcelona

08193 - Bellaterra, Barcelona

SPAIN

E-mail address: jllibre@mat.uab.es

Department of Mathematics

SHANGHAi JiAOTONG UNIVERSITY

Shanghai 200030

P.R. CHINA

E-mail address: xzhang@pine.njnu.edu.cn 